\title{
Periodicity of Strong Seismicity in Italy: Schuster Spectrum Analysis Extended to the Destructive Earthquakes of 2016
}

P. L. BRAGATO

\begin{abstract}
The strong earthquakes that occurred in Italy between 2009 and 2016 represent an abrupt acceleration of seismicity in respect of the previous 30 years. Such behavior seems to agree with the periodic rate change I observed in a previous paper. The present work improves that study by extending the data set up to the end of 2016, adopting the latest version of the historical seismic catalog of Italy, and introducing Schuster spectrum analysis for the detection of the oscillatory period and the assessment of its statistical significance. Applied to the declustered catalog of $M_{\mathrm{w}} \geq 6$ earthquakes that occurred between 1600 and 2016, the analysis individuates a marked periodicity of 46 years, which is recognized above the 95\% confidence level. Monte Carlo simulation shows that the oscillatory behavior is stable in respect of random errors on magnitude estimation. A parametric oscillatory model for the annual rate of seismicity is estimated by likelihood maximization under the hypothesis of inhomogeneous Poisson point process. According to the Akaike Information Criterion, such model outperforms the simpler homogeneous one with constant annual rate. A further element emerges form the analysis: so far, despite recent earthquakes, the Italian seismicity is still within a long-term decreasing trend established since the first half of the twentieth century.
\end{abstract}

Key words: Italian seismicity, periodicity, Schuster spectrum.

\section{Introduction}

In less than 8 years, between 2009 and 2016, Italy was struck by three seismic sequences with $M_{\mathrm{w}} \geq 6$ earthquakes that caused a large number of victims and heavy damage: the L'Aquila earthquake of April 6, $2009\left(M_{\mathrm{w}} 6.3,309\right.$ casualties); the Emilia earthquake of May 20, $2012\left(M_{\mathrm{w}} 6.1,27\right.$ casualties, including those of the main aftershock of 29 May) and the seismic sequence of 2016 in Central Italy,

1 Centro di Ricerche Sismologiche, Istituto Nazionale di Oceanografia e di Geofisica Sperimentale-OGS, Via Treviso 55, 33100 Udine (UD), Italy. E-mail: pbragato@inogs.it that started with an $M_{\mathrm{w}} 6.2$ earthquake on 24 August (299 casualties) and included two other strong earthquakes (26 October, $M_{\mathrm{w}} 6.1$, and 30 October, $\left.M_{\mathrm{w}} 6.5\right)$ that largely increased the damage, although with no further victims. The occurrence of such events represents a sudden acceleration of seismic activity over the last 30 years: in fact, after two strong earthquakes on 1976 (Friuli earthquake, $M_{\mathrm{w}}$ 6.5) and 1980 (Irpinia earthquake, $M_{\mathrm{w}}$ 6.8), a magnitude near to 6 was reached just one time, for the Umbria-Marche sequence of September 1997. In a previous study (Bragato 2015), I found that similar accelerations occur cyclically in the seismic history of Italy, more clearly since the seventeenth century. The strong social and economical impact of the earthquakes of 2016 in Central Italy led me to update that analysis and assess the strength of the periodicity. Other to extend the time of observation, the present study improves that of 2015 in three main aspects: first, it exploits the latest version of the historical seismic catalog of Italy, which revises location/magnitude estimations and guarantees homogeneous processing for events up to 2014 (2006 in the previous version); second, a more formal assessment of the periodicity and of its statistical significance is introduced, based on Schuster spectrum analysis (Ader and Avouac 2013); third, the robustness of the results is explored, considering perturbations of the historical seismic catalog obtained by altering the values of magnitude according to the corresponding estimation error and adopting different choice for declustering.

According to previous analyses (Bragato 2014; Bragato and Sugan 2014), the last century is characterized by the decrease in major seismicity in Italy. Furthermore, (Bragato 2017) poses such reduction at 
the end of a seismic transient originated in the seventeenth century. That result is here updated considering the earthquakes of 2016 and checking the persistence of the trend both in terms of number of mainshocks and of the cumulative released energy.

\section{Data Selection}

This study is based on the most recent version of the Catalogo Parametrico dei Terremoti Italiani (CPTI15, release 1.5, Rovida et al. 2016), an historical seismic catalog that includes 4584 earthquakes that occurred between the years 1000 and 2014, located in Italy and the surrounding area (those earthquakes that could affect the estimation of the seismic hazard in the country). The catalog is not declustered and contains the known mainshocks as well as a number of strong aftershocks. For each earthquake, it reports a value of moment magnitude $M_{\mathrm{w}}$ either estimated instrumentally or derived from observations of macroseismic intensities (Rovida et al. 2016). Each value of magnitude is provided with an estimation error that depends on the availability of data and on the type of computation (from about 0.5 in the historical part to about 0.1 in the instrumental era). I completed the catalog with data up to the end of 2016 drawn from the EuropeanMediterranean Regional Centroid Moment Tensor (RCMT) Catalog (Pondrelli et al. 2011). I used RCMT solutions for continuity with the past, because they are the main source of $M_{\mathrm{w}}$ in CPTI15 since 1976. The mixing of instrumental and macroseismic data is critical and could affect the comparison of the earthquake rates in different time periods. To mitigate the problem, the authors of the catalog estimated the magnitude of the historical earthquakes using the
Boxer method (Gasperini et al. 1999), a robust algorithm that takes into account the entire macroseismic field instead of the single value of maximum or epicentral intensity. Also the attribution of the site intensities (which is at the basis of $M_{\mathrm{w}}$ estimation) is extremely consistent through the centuries, because of the existence in Italy of a large number of historical residential buildings. In (Bragato 2017), I furnished partial evidence against a magnitude bias: analyzing the rate changes of the last century, I obtained almost identical results selecting the earthquakes by either magnitude or intensity threshold ( $M_{\mathrm{w}} \geq 4.8$ and $I_{\max } \geq$ VII, respectively).

For the completeness of the catalog, I referred to the estimations by Stucchi et al. (2011), based on the continuity of the historical documentary sources. In their Table 2, they report the magnitude of completeness for a number of seismic zones in different time periods. After 1600, the magnitude of completeness ranges between 5.91 and 6.14. I performed the analysis for the intermediated threshold $M_{\mathrm{w}} \geq 6$ [the same value used in (Bragato 2015)] and then confirmed the results for $M_{\mathrm{w}} \geq 5.8$ and $M_{\mathrm{w}} \geq 6.2$.

I mainly worked with the catalog declustered for the aftershocks. For simplicity, I carried out declustering using the method by Gardner and Knopoff (1974) (GK declustering hereafter) in its original formulation (i.e., using the magnitude-dependent space-time windows of their Table 1). For a sensitivity analysis, I also took in consideration the original catalog (not declustered) and that obtained by overdeclustering (i.e., using the GK space-time windows multiplied by 1.5 ).

After the selection by GK declustering and completeness level, the resulting catalog includes 60 earthquakes in the magnitude range 6.00-7.32 distributed in space according to Fig. 1.

Table 1

Maximum likelihood estimation (MLE) of parameters for different models of the earthquake rate function

\begin{tabular}{lllrr}
\hline Rate function $\lambda(t)$ & MLE parameters & $N$ & $k$ & $\ln \hat{L}$ \\
\hline$\lambda$ & $\lambda=0.14$ & 60 & 1 & -176.3 \\
$a+b \cos \left[2 \pi\left(t-t_{0}\right) / T\right]$ & $a=0.14, b=0.08$, & 60 & 4 & -171.0 \\
$\exp \left(a+b t+c t^{2}+d \cos \left[2 \pi\left(t-t_{0}\right) / T\right]\right)$ & $T=46.73, t_{0}=1599.28$ & & 354.7 \\
& $a=-2.38, b=4.8 \times 10^{-3}$, & 60 & 6 & -169.8 \\
& $c=-1.1 \times 10^{-5}, d=0.62$, & & \\
& $T=46.48, t_{0}=1600.19$ & & \\
\hline
\end{tabular}




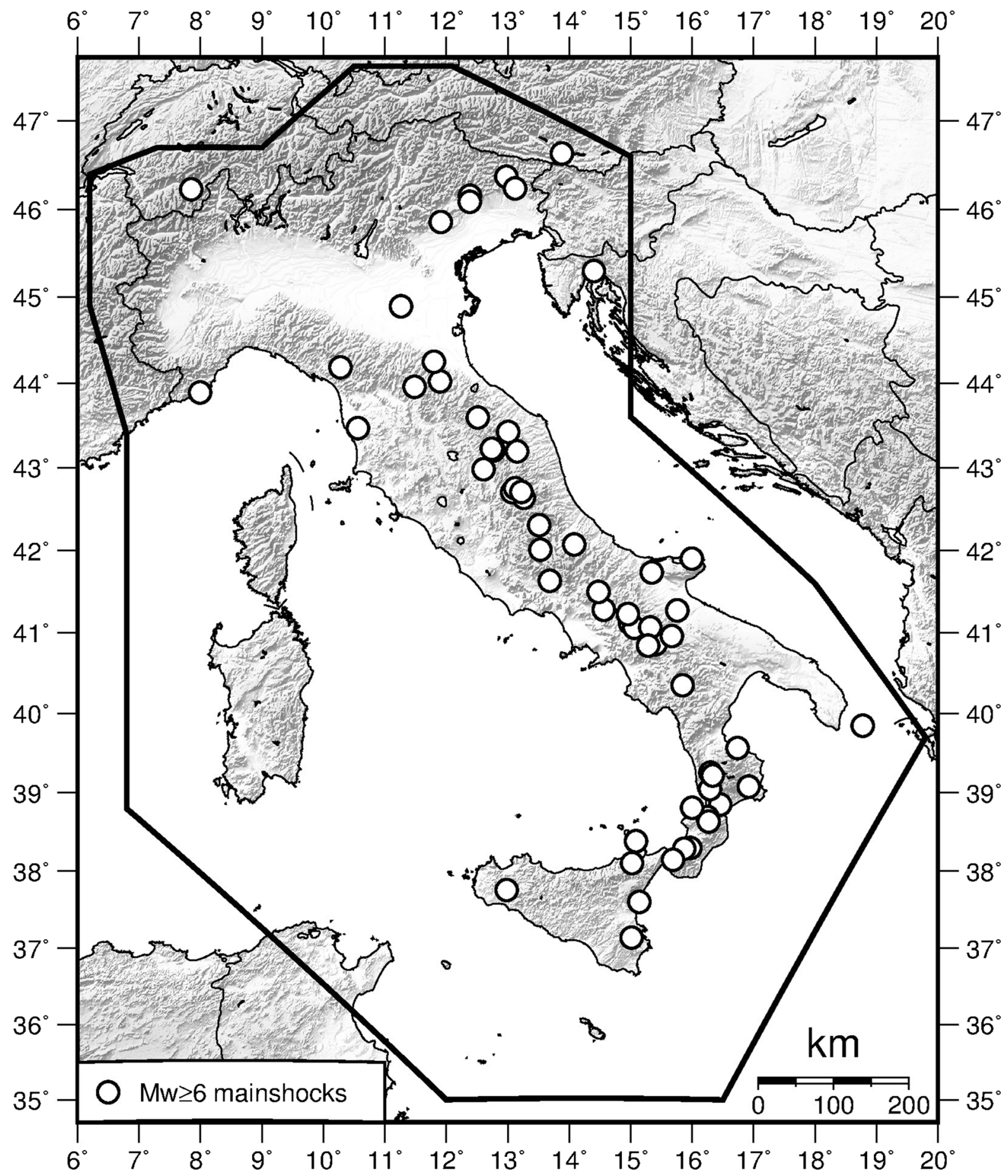

Figure 1

Areal coverage of the CPTI15 catalog (polygon) and epicenters of $M_{\mathrm{w}} \geq 6.0$ mainshocks that occurred in Italy and surroundings between 1600 and 2016 (circles) 


\section{Analysis}

I evaluated the periodicity of the earthquakes in two steps. First, I explored their time characteristics visually, tracing their smoothed time density. Second, I assessed the statistical significance and the robustness of the observed features by formal tests and Monte Carlo simulation. Figure 2a reports the variable annual rate of earthquakes estimated by kernel smoothing (Bowman and Azzalini 1997), according to

$$
\lambda(t)=\sum_{i=1}^{N} \varphi\left(t_{i}-t ; h\right),
$$

where $t_{1}, \ldots, t_{N}$ are the times of occurrence of the events, $\varphi(z ; h)$ is the kernel function (zero-mean normal density function in $z$ with standard deviation $h$ ), and $h$ is the smoothing parameter (the larger $h$, the larger the degree of smoothing). In Fig. 2a, I traced the curves obtained for $h=7$ years (coincident with the average interarrival time of the earthquakes) and $h=40$ years. I chose the two parameters arbitrary, with the aim to evidence both short-term and longterm details. The curve for $h=7$ years (thin line) has an almost regular oscillatory trend, with a major peak around 1700, four similar intermediate peaks between 1730 and 1930, and minor peaks at the beginning and at the end of the study period. The reduction of activity at the two extremes (about 50\%) is better evidenced by the smoothed curve traced for $h=40$ years (thick line in Fig. 2a). The long-term behavior is the subject of a previous study (Bragato 2017), where I showed the occurrence of a seismic transient between the seventeenth and twentieth centuries.

For a statistical characterization of the short-term oscillations of Fig. 2a, I analyzed the Schuster spectrum of the catalog according to (Ader and Avouac 2013). The method is based on the Schuster's test (Schuster 1897), originally introduced to explore the tidal modulation of earthquakes: to verify the possible periodicity at the time period $T$, the $k$-th earthquake in the catalog occurring at time $t_{k}$ is represented as a unit step in the direction defined by the phase angle $\theta_{k}=2 \pi t_{k} / T$. After $N$ steps ( $N$ the number of earthquakes in the catalog), the distance from the origin is given by $D^{2}=\left(\sum_{k=1}^{N} \cos \theta_{k}\right)^{2}+$ $\left(\sum_{k=1}^{N} \sin \theta_{k}\right)^{2}$. Under the hypothesis of a random uniform distribution of the earthquakes, the probability of observing a distance $D$ is $p=e^{-D^{2} / N}$. If $p<\alpha$, the hypothesis of uniform distribution of the events is rejected at the confidence level $(1-\alpha)$. Ader and Avouac (2013) observe that such condition is necessary but not sufficient to establish the
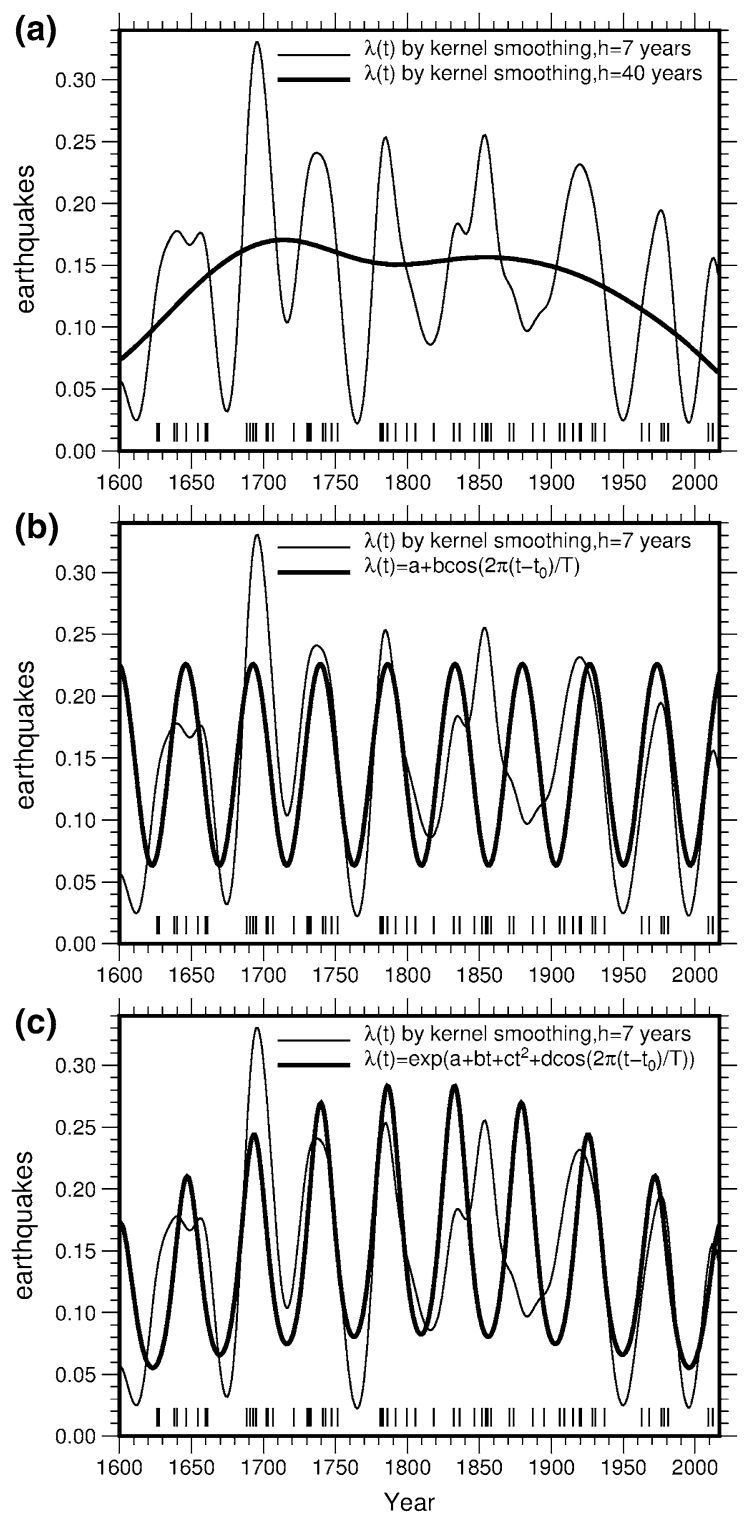

Figure 2

Rate function $\lambda(t)$ estimated for the declustered catalog of $M_{\mathrm{w}} \geq 6.0$ earthquakes using parametric (MLE) and nonparametric (kernel smoothing) models. The ticks at the bottom indicate the time of occurrence of the 60 earthquakes 
periodicity at period $T$. In fact, the non-uniformity detected by the Schuster test at the period $T$ can be induced also by a short and intense burst of seismicity occurring at any time. The authors suggest to analyze the entire Schuster spectrum (i.e., the values of $p$ computed for a range of time periods $T_{1}<T_{2}<\cdots<T_{\mathrm{m}}$ ): a significant sinusoidal periodicity corresponds to a narrow maximum in the spectrum. They also pose stricter limits for the probability $p$ in order to guarantee a given confidence level. They introduce $p$ thresholds that depend on the investigated oscillation period $T$ : a periodicity can be claimed to be detected above the $(1-\alpha)$ confidence level if $p<\alpha T / T_{\text {tot }}$ ( $T_{\text {tot }}$ the overall observation period) instead of $p<\alpha$, as required by the original test. I computed the Schuster spectrum for the declustered catalog in the time range 1600-2016, exploring periods of oscillation between 10 and 200 years (Fig. 3). The spectrum indicates a marked periodicity at 46 years, which is significant at the $95 \%$ confidence level. Assuming a sinusoidal oscillation around an average annual rate, such confidence level corresponds to oscillations with amplitude that is at least $52 \%$ of the average rate [Eq. 10 of (Ader and Avouac 2013)]. Figure 4 shows the distribution of the phase angles of the 60 earthquakes computed for

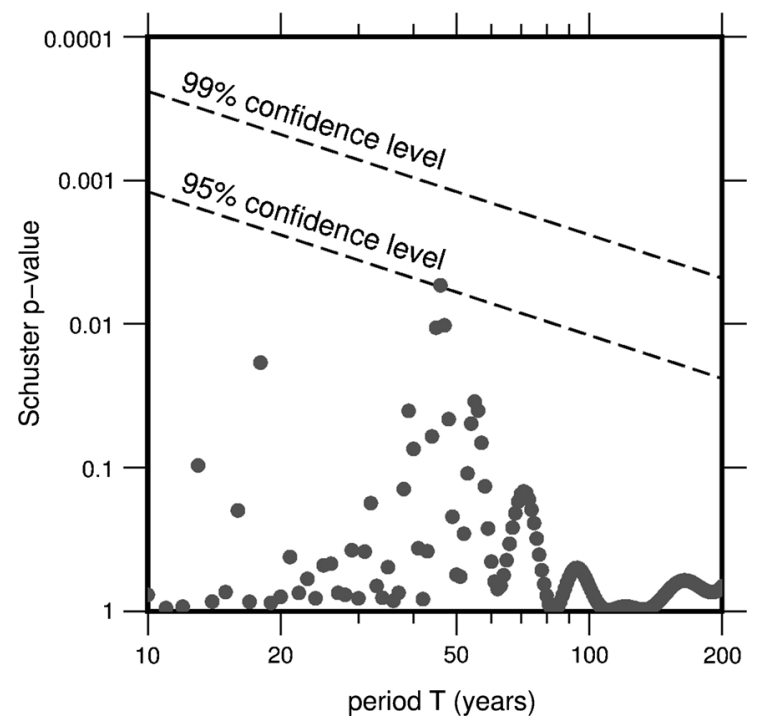

Figure 3

Schuster spectrum for the declustered catalog of $M_{\mathrm{w}} \geq 6.0$ earthquakes that occurred in Italy between 1600 and 2016

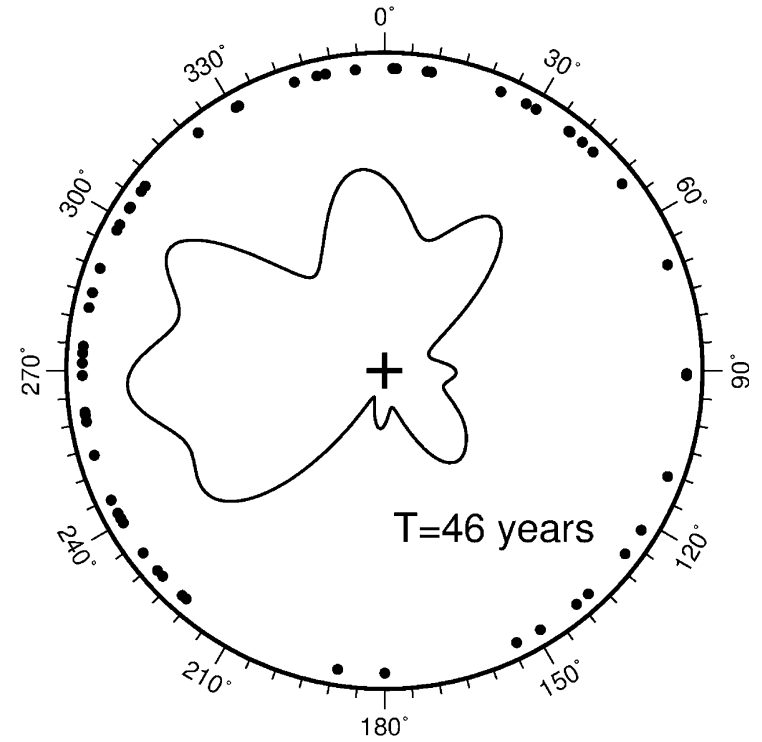

Figure 4

Phase angles corresponding to the period $T=46$ years computed for the $M_{\mathrm{w}} \geq 6.0$ earthquakes in the declustered catalog 1600-2016 (dots). The curve represents the density of earthquakes per degree estimated by kernel smoothing for $h=10^{\circ}$

$T=46$ years: it is clearly far from uniform, with a concentration on half of the circle.

One of the reviewers of the present paper pointed out a limit of the Shuster spectrum approach: increasing the number of periods $T$ for which the Schuster test is performed makes it easier to get a low $p$ value (in at least one test) just by chance. This is a particular instance of the "multiple testing problem" which, in general, requires a further reduction of the $p$ thresholds for significance (e.g., Aickin and Gensler 1996). Ader and Avouac (2013) do not consider the multiple testing problem. Based also on such limitation, it appears useful to assess the significance of the periodicity in an alternative way. I chose to adopt a technique of model selection inference (Imoto et al. 1999; Lee et al. 1991): the hypothesis of a Poisson point process having constant rate $\lambda$ is compared with that of a Poisson point process with a time-dependent rate that includes a cyclic component. I explored the use of a rate function of type:

$$
\lambda\left(t, a, b, t_{0}, T\right)=a+b \cos \left[2 \pi\left(t-t_{0}\right) / T\right],
$$

where the average rate $a$ is summed to an oscillation of amplitude $b$, period $T$ and initial shift $t_{0}$. I obtained 
the best-fitting parameters of the two models ( $\lambda$ in the constant-rate case, $a, b, T$ and $t_{0}$ in the other case) by maximum likelihood estimation (MLE). For the generic Poisson point process with parametric rate function $\lambda(t, \theta)$ ( $\theta$ the vector of parameters), the likelihood has equation:

$$
L\left(t_{1}, t_{2}, \ldots, t_{N}, \theta\right)=e^{-\int_{A}^{B} \lambda(t, \theta) \mathrm{d} t} \prod_{i=1}^{N} \lambda\left(t_{i}, \theta\right),
$$

where $t_{1}, \ldots, t_{N}$ are the arrival times in the interval $(A$, $B)$. For the constant-rate process, the estimation reduces to $\lambda=N /(B-A)$, while for the oscillatory rate function the maximization must be performed by numerical search. The estimated parameters and the corresponding log-likelihood are reported in Table 1 . Thanks to the use of a larger number of parameters, the oscillatory model reaches a higher likelihood, which testifies a better fit. The significance of the improvement can be judged by means of the Akaike Information Criterion [AIC, Akaike (1977)]: the use of the more complex model of Eq. 2 is justified if it reduces the quantity

$$
\operatorname{AIC}(\hat{L}, k)=-2 \ln \hat{L}+2 k,
$$

( $\hat{L}$ the likelihood estimated for the model, $k$ the number of parameters) in respect of the same quantity computed for the constant-rate model. Moreover, in the present case, since the number of data points $N$ is low compared to the number of parameters $k[N /$ $k<40$ according to (Burnham and Anderson 2002)], a correction for small samples is introduced, leading to the second-order AIC:

$$
\begin{aligned}
\operatorname{AICc}(\hat{L}, k, N)= & -2 \ln \hat{L}+2 k \\
& +2 k(k+1) /(N-k-1) .
\end{aligned}
$$

For the declustered sequence of $M_{\mathrm{w}} \geq 6$ earthquakes in Italy, the oscillatory model (Fig. 2b, Table 1) has the lowest AICc and is preferable to the constant-rate model. The difference of the two values of AICc (4.0) is indicative of the quality of the worst model (that with constant rate in the present case) compared to the best one: the larger the difference, the lower the quality. As a rule of thumb (Burnham and Anderson 2002), a difference between 4 and 7 indicates that the worst model has considerably less support than the best one, although it cannot be completely discarded (it is judged very unlikely if the difference is larger than 10). It is to note that the sinusoidal model converges to an oscillation period near to that obtained by Schuster spectrum analysis (46.73 years vs. 46). Also the percentage of oscillation in respect of the average rate is very similar: $57 \%$ (ratio $b / a$ in Eq. 2) compared to $52 \%$ from spectrum analysis.

In order to capture also the long-term trend of Fig. 2a (thick line), I tried a model combining the oscillatory component with a quadratic term:

$$
\begin{aligned}
& \lambda\left(t, a, b, c, d, t_{0}, T\right)=\exp \left(a+b t+c t^{2}\right), \\
& +d \cos \left[2 \pi\left(t-t_{0}\right) / T\right]
\end{aligned}
$$

where the exponential guarantees the condition $\lambda(t)>0$ [this class of models is discussed by Lee et al. (1991)]. The use of Eq. 6 (Fig. 2c, Table 1) increases the likelihood but, because of the higher number of parameters (6 instead of 4$)$, the model is not preferred by the AICc. This result indicates that the long-term trend, although present, is too short and weak to gain a statistical significance. Nonetheless, the decrease of the last century is important for hazard assessment. This period can be investigated with more details thanks to the completeness of the catalog at a lower magnitude threshold. In previous studies, I analyzed such trend at different space scales, both in Italy (Bragato 2014, 2017) and globally (Bragato and Sugan 2014). In particular, in (Bragato 2017) I showed the statistical significance of the decrease for $M_{\mathrm{w}} \geq 4.8$ earthquakes that occurred in Italy between 1900 and 2015. The finding remains unchanged after the extension of the catalog to 2016. The decrease can also be evidenced looking at the energy radiated by the earthquakes, computed according to the equation $\log \left(E_{\mathrm{S}}\right)=1.5 M_{\mathrm{w}}+11.8$ (Gutenberg and Richter 1956). The graph of Fig. 5 shows the cumulative energy computed for $M \geq 4.8$ earthquakes in the catalog (both original and declustered) since 1900. The three major steps appear at the beginning of the twentieth century, around 1980 and in the last decade. The progressive reduction of the amplitude of the step indicates that at present, despite the short-term acceleration, the seismic behavior is still within a secular decreasing trend.

For a graphical appraisal of the quality of the fit by the parametric models, in Fig. $2 b$, c they are 


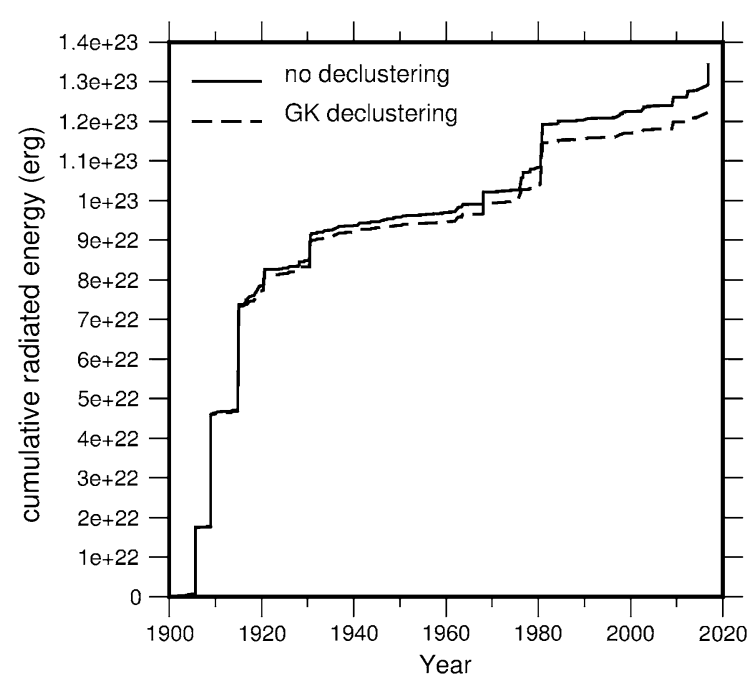

Figure 5

Cumulative radiated energy of $M_{\mathrm{w}} \geq 4.8$ earthquakes in Italy since 1900 computed for the original and the declustered catalogs

compared to the nonparametric one (Eq. 1, computed for $h=7$ years) that, thanks to the higher flexibility, gives a better representation of the underlying data distribution. Since the occurrence of the first earthquake on 1626, the curves are in good agreement on 7 of the 9 cycles. The quadratic-oscillatory curve (Fig. 2c) improves the fitting of the last three cycles and, in particular, matches very well the acceleration of the last decade. Both graphs show major differences in the nineteenth century. There, the behavior can be split in a smaller peak of activity around 1830, in agreement with the periodic oscillation, and a denser cluster of earthquakes centered on 1855, appearing 20 years in advance compared to the theoretical cycle. Such cluster represents an outlier in an otherwise regular trend.

I performed a Monte Carlo simulation to assess the strength and the stability of the periodicity in respect of errors in magnitude estimation. I generated 1,000 alternative catalogs taking the original catalog (prior to declustering and to the selection for $M_{\mathrm{w}} \geq 6$ ), replacing each value of magnitude $M_{i}$ with a random value drawn from a normal distribution $N\left(M_{i}, \sigma_{M i}\right)\left(\sigma_{M i}\right.$ the magnitude error reported in the catalog), and then performing GK declustering and the selection for $M_{\mathrm{w}} \geq 6$. For each perturbed catalog, I estimated the smoothed density, which is shown as a gray line in Fig. 6. The figure also reports the 16th, 50th and 86th percentile of the perturbed densities (dashed lines), which are compared with the smoothed density of the original catalog (black continuous line). A prominent feature of Fig. 6 is that the perturbed densities are not centered on that of the original catalog, but biased toward larger values. This is an effect of the Gutenberg-Richter distribution of earthquakes: there are more earthquakes of low magnitude that, after perturbation, go over the magnitude threshold, than earthquakes of high magnitude that go under the threshold. One could try to correct such bias, for example changing the distribution of the errors and assuming that it is not normal, which is in turn a rather controversial hypothesis. I did not elaborate further. I only observe that even with this distortion, the overall set of perturbed time densities in Fig. 6 maintains the oscillatory character of the original distribution. For each perturbed catalog, I performed Schuster spectrum analysis: in the 1000 cases, the estimated period of oscillation ranges from 35 to 57 years, with an average value of 45.9 years (46 years for the original catalog). The average

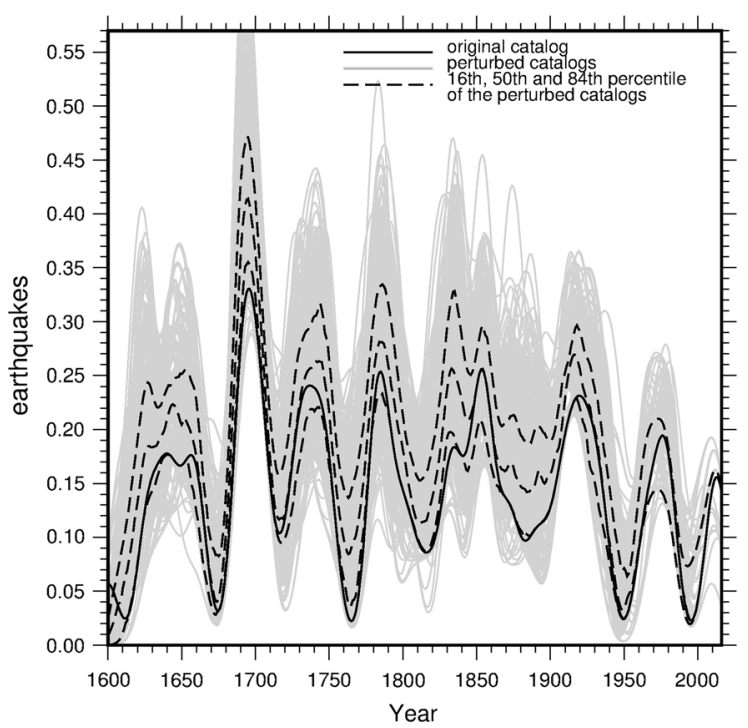

Figure 6

Rate functions $\lambda(t)$ estimated by kernel smoothing ( $h=7$ years) for 1000 catalogs obtained from the original one by perturbing the values of magnitude according to the associated estimation error (gray lines). The catalogs were GK declustered and cut at the threshold $M_{\mathrm{w}} \geq 6.0$. The dashed lines indicate the 16th, 50th and 84th percentile of the perturbed rate functions. The continuous line is the rate function computed for the original (not perturbed) declustered catalog 
confidence level of the detection is $92.1 \%$, while it is greater than $95 \%$ for 588 catalogs. Similarly, for each perturbed catalog I estimated the parametric model of Eq. 2 and carried out AICc computation to check whether it performs better than the constant-rate $\lambda$ model. For the largest part of the catalogs (89\%), the oscillatory model has lowest AICc. On average, the difference of the two AICc is 3.7 (4.0 for the original catalog). The estimation of the oscillatory period $\mathrm{T}$ for the perturbed catalogs ranges from 43.9 to 48.5 years, with an average value of 46.9 years.

To assess the sensitivity to declustering, I also tried no declustering and overdeclustering (i.e., using the GK space/time windows for aftershock selection multiplied by 1.5). The number of events is 67 and 57 for no declustering and overdeclustering, respectively (compared to 60 for standard GK declustering). In the two cases (dotted and dashed lines in Fig. 7), the effect is that of enhancing or reducing the peaks, leaving the period of the oscillation unchanged. In particular, major differences between GK declustering and no declustering appear in correspondence with four peaks, corresponding to seismic sequences with two or more $M_{\mathrm{w}} \geq 6$ earthquakes. This fact indicates that sequences like that of 2016,

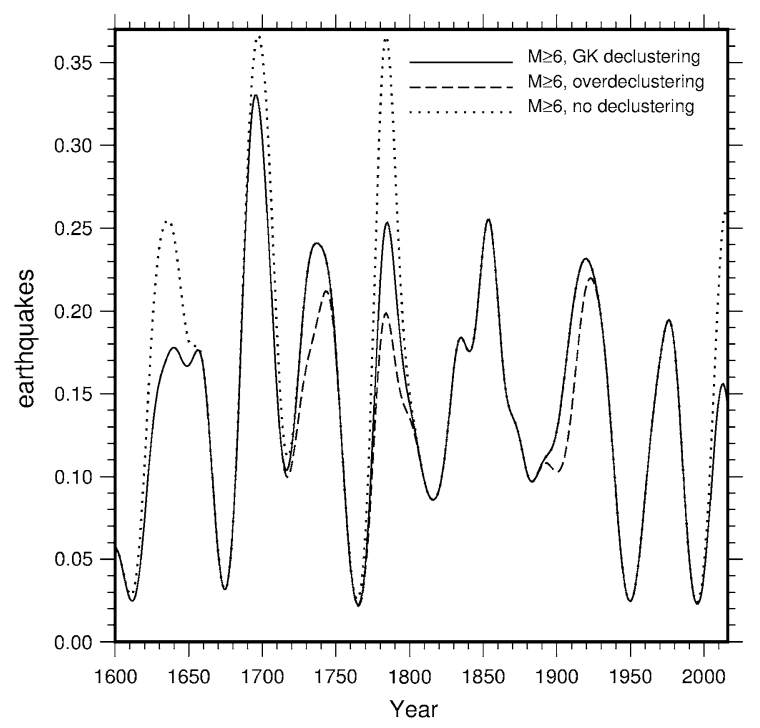

Figure 7

Smoothed rate function ( $h=7$ years) of $M_{\mathrm{w}} \geq 6.0$ earthquakes that occurred in Italy since 1600 and selected according to different types of declustering (GK, overdeclustering and no declustering) characterized by series of strong earthquakes, are rather common in Italy.

The last sensitivity test I performed concerns the cut of the catalog at different magnitude thresholds. In Fig. 8, I compared the annual rate of the mainshocks selected for $M_{\mathrm{w}} \geq 6.2, \quad M_{\mathrm{w}} \geq 6.0$ and $M_{\mathrm{w}} \geq 5.8$ (42, 60 and 94 earthquakes, respectively). In particular, the latter value is under the magnitude of completeness and the corresponding annual rate should give an indication of the robustness of the periodicity against the loss of some earthquakes. The three curves in Fig. 8 share the same oscillatory character, although with some differences in the relative amplitude of the peaks.

\section{Discussion and Conclusions}

The earthquakes of the last decade confirm the oscillatory character of strong seismicity in Italy, with time clusters that spread over large areas including different tectonic domains. The fluctuations are superimposed to a long-term trend that for the last century is decreasing. The period of the oscillation estimated in the present work differs from that of

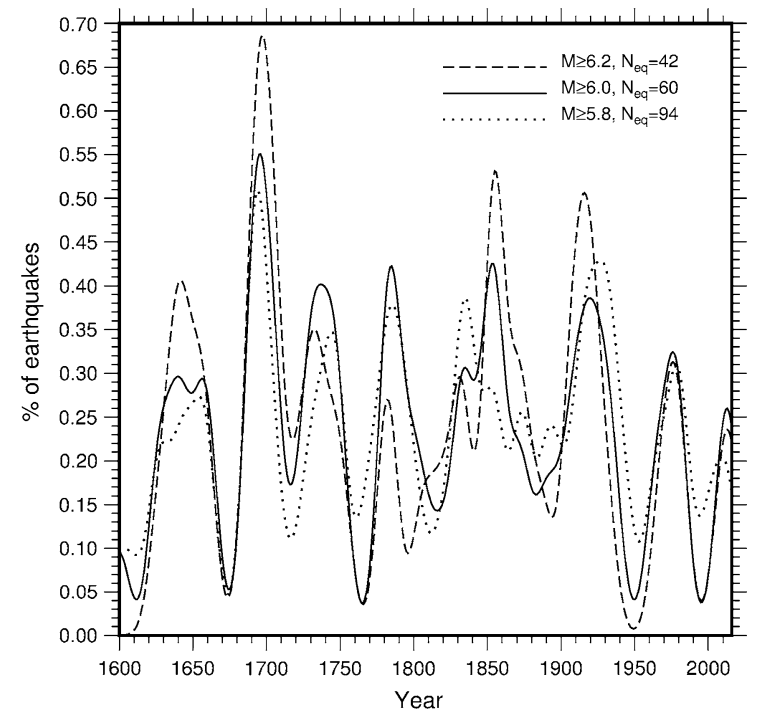

Figure 8

Smoothed rate function ( $h=7$ years) of the mainshocks that occurred in Italy since 1600 and selected according to different values of the minimum magnitude. For a better comparison, the rate is normalized to the total number of earthquakes in each catalog $\left(100 \lambda(t) / N_{\text {eq }}\right)$ 
(Bragato 2015), reducing from 55 to 46 years. The previous assessment converged to a solution maximizing the fit to the cluster centered on 1855 . With the revision of the catalog and its extension to 2016, the relative weight of that cluster is reduced, so that now it is seen as an outlier and skipped by the oscillatory models (Fig. 2b, c).

The observed cyclic behavior is important for time-dependent seismic hazard analysis. It suggests that the return period of major earthquakes must be searched and analyzed at the national/regional scale other than locally (i.e., for single faults or systems of connected faults). My analysis is someway related to previous works on the clustering of strong earthquakes in Italy (Faenza et al. 2003, 2004; Lombardi and Marzocchi 2009). Those studies use much more complex statistical models that take into account both time and spatial characteristics of seismicity. They show that the strong earthquakes are characterized by a significant degree of time clustering. Furthermore, the duration of such clusters is much longer than that expected for aftershocks sequences, suggesting a different physical mechanism of interaction. In particular, Lombardi and Marzocchi (2009) estimate an average relaxation time $\tau$ of about 30 years, comparable with the length of a half cycle of increased activity in Fig. 2 (23 years). My results confirm existence, significance and duration of the time clusters, with the further indication of their periodicity.

This work is limited to the particular case of Italy, exploiting the availability of a high-quality historical catalog. It seems plausible that the periodicity extends to a larger area (at least that sharing the same tectonic environment of Italy). An investigation in this direction is prevented by the heterogeneity of the historical seismic catalogs available for different countries. In Europe, the SHARE European Earthquake Catalog (Stucchi et al. 2012) represents an attempt to revise and integrate the various catalogs adopting common standards, but the quality of the overall common catalog is far from being comparable with that of the Italian one (especially for what concerns the minimum magnitude of completeness). Nonetheless, a few studies (Mantovani and Albarello 1997; Viti et al. 2003; Mantovani et al. 2010) have found some degree of synchronization (although with no periodicity) between the strong earthquakes occurring on the two sides of the Adriatic Sea, in correspondence with the borders of the Adriatic microplate (Southern Apennines on west and Southern Dinarides-Albanides on east).

At present, a physical explanation for the seismic periodicity can be only conjectured. A number of alternative hypotheses are discussed in (Bragato 2015) with reference to the available literature. They include the mutual triggering of earthquakes through different mechanisms of stress transfer, the existence of tectonic pulses as well as the control of seismicity by processes that are external to the geosphere. In particular, I focused on climate-related surface processes involving the redistribution of large masses (ice and water). Various papers have shown that despite the weak stress changes they can cause at depth compared to the tectonic stress, they are potentially able to control seismicity at different time scales: from seasonal (Bettinelli et al. 2008) to millennial (Luttrell and Sandwell 2010; Hampel et al. 2010). Furthermore, some climate processes have a periodicity similar to that of the earthquakes (Scafetta and Mazzarella 2015). In (Bragato 2017), I extended the analysis back in time from 1600 to 1100 . I considered a reconstruction of the history of the global surface temperature (Mann et al. 2008) as a proxy for that of the sea level. Using binomial logistic regression, I found that the occurrence of the earthquakes is significantly anticorrelated with the surface temperature with a delay of 174 years. In particular, there is good correspondence between the occurrence of the Little Ice Age (fifteenth-eighteenth centuries) and that of a seismic transient in Italy (seventeenthtwentieth centuries). According to that analysis, the recent acceleration of seismicity (years 2009-2016) should be correlated with a cold period that occurred in the first half of the nineteenth century.

\section{Data and Resources}

The seismic catalog CPTI15 (Rovida et al. 2016) was downloaded from the web site http://emidius.mi. ingv.it/CPTI (last accessed March 2017). The European-Mediterranean Regional Centroid Moment Tensor (RCMT) Catalog (Pondrelli et al. 2011) was 
available online at http://www.bo.ingv.it/RCMT (last accessed March 2017). All the figures were produced using the Generic Mapping Tool version 5.1.1 (Wessel et al. 2013) available at http://www.soest. hawaii.edu/gmt (last accessed March 2016). Part of the statistical analysis was performed using the $\mathrm{R}$ software system (R Core Team 2012).

\section{Acknowledgements}

This research was supported by Regione Friuli Venezia Giulia and Regione Veneto. I thank the Editor, Eric Geist, and two anonymous reviewers for reading the paper and suggesting useful improvements.

Open Access This article is distributed under the terms of the Creative Commons Attribution 4.0 International License (http:// creativecommons.org/licenses/by/4.0/), which permits unrestricted use, distribution, and reproduction in any medium, provided you give appropriate credit to the original author(s) and the source, provide a link to the Creative Commons license, and indicate if changes were made.

\section{REFERENCES}

Ader, T. J., \& Avouac, J. P. (2013). Detecting periodicities and declustering in earthquake catalogs using the Schuster spectrum, application to Himalayan seismicity. Earth and Planetary Science Letters, 377-378, 97-105. doi:10.1016/j.eps1.2013.06.032.

Aickin, M., \& Gensler, H. (1996). Adjusting for multiple testing when reporting research results: the Bonferroni vs Holm methods. American Journal of Public Health, 86, 726-728.

Akaike, H. (1977). On entropy maximization principle. In P. R. Krishnaiah (Ed.), Proceedings of the symposium on applications of statistics (pp. 27-47). North-Holland: Amsterdam.

Bettinelli, P., Avouac, J. P., Flouzat, M., Bollinger, L., Ramillien, G., Rajaure, S., et al. (2008). Seasonal variations of seismicity and geodetic strain in the Himalaya induced by surface hydrology. Earth Planet Science Letters, 266, 332-344. doi:10.1016/j. eps1.2007.11.021.

Bowman, A. W., \& Azzalini, A. (1997). Applied smoothing techniques for data analysis. Oxford: Oxford University Press.

Bragato, P. L. (2014). Rate changes, premonitory quiescence, and synchronization of earthquakes in northern Italy and its surroundings. Seismological Research Letters, 85, 639-648. doi:10. 1785/0220130139.

Bragato, P. L. (2015). Italian seismicity and Vesuvius' eruptions synchronize on a quasi 60-year oscillation. Earth and Space Science, 2, 134-143. doi:10.1002/2014EA000030.

Bragato, P. L. (2017). A statistical investigation on a seismic transient occurred in Italy between the 17th and the 20th centuries. Pure and Applied Geophysics, 174, 907-923. doi:10. 1007/s00024-016-1429-2.

Bragato, P. L., \& Sugan, M. (2014). Decreasing rate of $M \geq 7$ earthquakes in the northern hemisphere since 1900. Seismological Research Letters, 85, 1234-1242. doi:10.1785/0220140111.

Burnham, K. P., \& Anderson, D. R. (2002). Model selection and multimodel inference: a practical information-theoretic approach (2nd ed.). New York: Springer.

Faenza, L., Marzocchi, W., \& Boschi, E. (2003). A non-parametric hazard model to characterize the spatio-temporal occurrence of large earthquakes; an application to the Italian catalogue. Geophysical Journal International, 155, 521-531. doi:10.1046/j. 1365-246X.2003.02068.x.

Faenza, L., Marzocchi, W., Lombardi, A. M., \& Console, R. (2004). Some insights into the time clustering of large earthquakes in Italy. Annals Geophysics, 47, 1635-1640.

Gardner, J. K., \& Knopoff, L. (1974). Is the sequence of earthquakes in Southern California, with aftershocks removed, Poissonian? Bulletin of the Seismological Society of America, 64, 1363-1367.

Gasperini, P., Bernardini, F., Valensise, G., \& Boschi, E. (1999). Defining seismogenic sources from historical earthquake felt reports. Bulletin of the Seismological Society of America, 89, 94-110.

Gutenberg, B., \& Richter, C. F. (1956). Magnitude and energy of earthquakes. Annali di Geofisica, 9, 1-15.

Hampel, A., Hetzel, R., \& Maniatis, G. (2010). Response of faults to climate-driven changes in ice and water volumes on earth's surface. Philosophical Transactions of the Royal Society of London A: Mathematical, Physical and Engineering Sciences, 368, 2501-2517. doi:10.1098/rsta.2010.0031.

Imoto, M., Maeda, K., \& Yoshida, A. (1999). Use of statistical models to analyze periodic seismicity observed for clusters in the Kanto region, central Japan. Pure and Applied Geophysics, 155, 609-624.

Lee, S., Wilson, J. R., \& Crawford, M. M. (1991). Modeling and simulation of a nonhomogeneous Poisson process having cyclic behavior. Communications in Statistics-Simulation and Computation, 20, 777-809.

Lombardi, A. M., \& Marzocchi, W. (2009). Double branching model to forecast the next $M \geq 5.5$ earthquakes in Italy. Tectonophysics, 475, 514-523. doi:10.1016/j.tecto.2009.06.014.

Luttrell, K., \& Sandwell, D. (2010). Ocean loading effects on stress at near shore plate boundary fault systems. Journal of Geophysical Research, 115, B08411. doi:10.1029/2009JB006541.

Mann, M. E., Zhang, Z., Hughes, M. K., Bradley, R. S., Miller, S. K., Rutherford, S., et al. (2008). Proxy-based reconstructions of hemispheric and global surface temperature variations over the past two millennia. Proceedings of the National academy of Sciences of the United States of America, 105, 13252-13257. doi:10.1073/pnas.0805721105.

Mantovani, E., \& Albarello, D. (1997). Medium-term precursors of strong earthquakes in southern Italy. Physics of the Earth and Planetary Interiors, 101, 49-60.

Mantovani, E., Viti, M., Babbucci, D., Albarello, D., Cenni, N., \& Vannucchi, A. (2010). Long-term earthquake triggering in the Southern and Northern Apennines. Journal of Seismology, 14, 53-65. doi:10.1007/s10950-008-9141-z.

Pondrelli, S., Salimbeni, S., Morelli, A., Ekström, G., Postpischl, L., Vannucci, G., et al. (2011). European-Mediterranean regional centroid moment tensor catalog: solutions for 2005-2008. 
Physics of the Earth and Planetary Interiors, 185, 74-81. doi:10. 1016/j.pepi.2011.01.007.

Rovida, A., Locati, M., Camassi, R., Lolli, B., \& Gasperini, P. (2016). CPTI15, the 2015 version of the parametric catalogue of Italian earthquakes. Istituto Nazionale di Geofisica e Vulcanologia. doi:10.6092/INGV.IT-CPTI15.

R Core Team (2012). R: a language and environment for statistical computing ( $\mathrm{R}$ Foundation for Statistical Computing, Vienna). http://www.r-project.org. Accessed July 2016.

Scafetta, N., \& Mazzarella, A. (2015). Spectral coherence between climate oscillations and the $M \geq 7$ earthquake historical worldwide record. Natural Hazards, 76, 1807-1829. doi:10.1007/ s11069-014-1571-z.

Schuster, A. (1897). On lunar and solar periodicities of earthquakes. Proceedings of the Royal Society of London, 61, 455-465.
Stucchi, M., Meletti, C., Montaldo, V., Crowley, H., Calvi, G. M., \& Boschi, E. (2011). Seismic hazard assessment (2003-2009) for the Italian building code. Bulletin of the Seismological Society of America, 101, 1885-1911. doi:10.1785/0120100130.

Stucchi, M., Rovida, A., Capera, A. A. G., Alexandre, P., Camelbeeck, T., Demircioglu, M. B., et al. (2012). The SHARE European earthquake catalogue (SHEEC) 1000-1899. Journal of Seismology, 17, 523-544. doi:10.1007/s10950-012-9335-2.

Viti, M., D’Onza, F., Mantovani, E., Albarello, D., \& Cenni, N. (2003). Post-seismic relaxation and earthquake triggering in the southern Adriatic region. Geophysical Journal International, 153, 645-657.

Wessel, P., Smith, W. H. F., Scharroo, R., Luis, J. F., \& Wobbe, F. (2013). Generic mapping tools: improved version released. Eos, Transactions American Geophysical Union, 94, 409-410. doi:10. 1002/2013EO450001.

(Received March 22, 2017, revised June 7, 2017, accepted June 9, 2017, Published online June 17, 2017) 